\title{
Flow-Induced Alignment of Block Copolymer-Sol Nanoparticle Coassemblies toward Oriented Bulk Polymer-Silica Hybrids
}

\author{
Anurag Jain, ${ }^{\dagger}$ Lisa M. Hall, ${ }^{\ddagger}$ Carlos B. W. Garcia, ${ }^{\dagger}$ Sol M. Gruner, ${ }^{\S}$ and \\ Ulrich Wiesner, ${ }^{*} \uparrow$
}

\begin{abstract}
Department of Materials Science and Engineering, Bard Hall, Cornell University, Ithaca, New York 14853; Rose-Hulman Institute of Technology, Terre Haute, Indiana; and Department of Physics and CHESS, Cornell University, Ithaca, New York
\end{abstract}

Received August 3, 2004; Revised Manuscript Received June 16, 2005

\begin{abstract}
Macroscopically oriented bulk organic-inorganic hybrid materials were cast from solutions subjected to hydrodynamic flow by utilizing a simple flow device. The hybrids were prepared from an amphiphilic diblock copolymer, poly(isoprene-block-ethylene oxide) (PI-b-PEO), used as a structure directing agent for organically modified ceramic precursors, (3-glycidyloxypropyl)trimethoxysilane (GLYMO) and aluminum sec-butoxide $\left(\mathrm{Al}\left(\mathrm{O}^{\mathrm{s}} \mathrm{Bu}\right)_{3}\right)$, prehydrolyzed into sol nanoparticles. Varying amounts of these ceramic precursors (sol) were used to obtain different mesoscale structures, i.e., lamellae, and the regular and inverse hexagonal mesophases. To align the microstructure, flow fields were applied during microstructure setting. To this end, a custom-built device operated in a Büchi evaporator subjected solutions of the polymer and ceramic precursors to a steady flow while the solvent evaporated. The flow induced a shearing force causing macroscopic alignment of the mesoscale structures. Small-angle X-ray scattering (SAXS) was used to characterize the oriented hybrids. From these data order parameters were calculated to quantify the degree of alignment in the hybrids in different directions. Order parameters as high as 0.77 were obtained for hybrids with lamellae lying parallel to the substrate. Alignment could also be evidenced in the hexagonal and inverse hexagonal samples with cylinder orientations along the flow direction. The results suggest the efficacy of this relatively simple flow technique in providing bulk polymer-inorganic hybrids with macroscopic alignment needed for realization of their true application potential.
\end{abstract}

\section{Introduction}

Microphase separation of block copolymers in periodic domains has opened novel avenues for generation of materials with tremendous control over shape and size at the nanoscale. Depending on the relative lengths of individual blocks, spheres, cylinders, bicontinuous cubic, or lamellar phases can be formed. ${ }^{1}$ Self-assembly in these systems therefore provides a versatile means to create nanostructured materials with properties relevant to such diverse fields as microelectronics and optics. However, inferior mechanical properties and a polycrystalline microstructure often severely limit the use of these soft materials in applications. Research in the field of nanocomposites has shown that nanoscale inorganic particles used at relatively small loadings impart considerable mechanical robustness to soft matter. ${ }^{2}$ Careful manipulation of the interface between the organic and inorganic species can be used to compatibilize these dissimilar and usually incompatible components.

At the beginning of the 1990s, using small organic amphiphiles, inorganic sol-gel silica-type components were shown to spontaneously coassemble into ordered composites. $^{3,4}$ More recently, this approach has been successfully extended to amphiphilic block copolymers providing access to mechanically robust polymerinorganic hybrid materials and mesoporous solids with novel physical properties, complex functions, and structural features. ${ }^{5-7}$ With local periodicities in the range of tens of nanometers, these structures are relevant for many potential applications such as size selective

\footnotetext{
Department of Materials Science and Engineering, Cornell University.

$\doteqdot$ Rose-Hulman Institute of Technology.

$\S$ Department of Physics, Cornell University.
}

separation, catalysis, chemical sensing, and photonic materials. $^{8}$

When the sol-gel precursors are hydrolyzed under moderately acidic conditions ( $\mathrm{pH}$ of $\sim 5$ ) before addition to the block copolymer dissolved in an organic solvent, the polymer-inorganic hybrid mesostructure formation mechanism upon solvent evaporation involves block copolymer-sol nanoparticle coassembly. ${ }^{6,9}$ We refer to this regime as the dense nanoparticle regime, in comparison to simulation work by Balazs et al. on block copolymer nanoparticle coassembly with relatively small particle volume fractions. ${ }^{9,10}$ The unusual degree of structure control observed in our system is thus very promising since the formation mechanism should be universally applicable to other block copolymer/inorganic nanoparticle assemblies provided that the nanoparticles are small enough and selectively compatible with only one of the blocks of the block copolymer. ${ }^{8,9}$

Despite tremendous potential, coassembly of block copolymers with inorganic materials leads to hierarchical order with phase-separated regions forming grains with sizes of the order of a few microns. Cylindrical or lamellar mesophases are directional, which results in local mechanical anisotropy in the grains of these materials. However, the grains are relatively small in comparison to the dimensions of the sample needed for usual applications, and often randomly distributed, which puts severe limitations on their utility. Apart from the large grain forming bicontinuous cubic hybrids, ${ }^{11,12}$ which are challenging to obtain experimentally because of their restricted window of stability, the more common phases require development of simple approaches for the generation of macroscopically aligned bulk samples for realization of the full application potential of these hybrid materials. 
Flow fields have been successfully used to change the structure and long-range order in pure block copolymers. ${ }^{1}$ Orientation of the microstructure both in the melt ${ }^{13-15}$ and in solution, ${ }^{16}$ either parallel or perpendicular to the direction of flow, can be achieved by carefully manipulating the flow fields and conditions. However, coassembly in polymer-sol nanoparticle hybrids is accompanied by inorganic nanoparticle gelation, preventing straightforward extension of these approaches to obtain macroscopically aligned nanostructured bulk hybrids. Alignment in such systems has therefore been obtained using surface effects, extensional flow, or considerable modification during the hybrid synthesis procedures. ${ }^{6,17-19}$ As an example, dip coating has been used to achieve high degrees of orientation in hybrid thin $(<1 \mu \mathrm{m})$ films obtained through evaporation induced self-assembly (EISA). ${ }^{17,20}$ In our first report on block copolymer directed aluminosilicate mesostructures, we reported that simple film casting leads to substantial macroscopic orientation of the lamellae parallel to the substrate. ${ }^{6}$ While these techniques clearly demonstrate the ability to achieve orientation effects in nanostructured silica-type hybrids, they are usually applicable only to thin films and/or not adaptable to large-scale synthesis of bulk materials. Here we present a straightforward scheme for the macroscopic alignment of bulk polymer-nanoparticle hybrid materials from solutions subjected to hydrodynamic flow based on a simplified version of the "rollcasting" method used by Albalak et al. ${ }^{21}$ to produce oriented pure block copolymer samples. The technique requires initially relatively low-viscosity starting solutions. This is advantageous for solutions, such as the examples discussed in this work, which are not viscous enough to work well with roll-casting. Also, this method may be extended in a straightforward way to a larger scale commercial process.

\section{Experimental Methods}

Synthesis. Two different poly(isoprene- $b$-ethylene oxide) (PI- $b$-PEO) amphiphilic diblock copolymers synthesized via anionic polymerization techniques were used in this study. ${ }^{22}$ Gel permeation chromatography (GPC) was employed to determine the precursor (polyisoprene) molecular weight and the dispersity of the block copolymers. ${ }^{1} \mathrm{H}$ NMR was used to determine the relative volume fractions of individual blocks and the microstructure of the PI blocks. The results were combined to determine the overall molecular weight of the block copolymers. The molecular parameters were determined to be $M_{\mathrm{n}}=19900 \mathrm{~g} / \mathrm{mol}$ for polymer P1 and 22700 for polymer P2. Both polymers have a volume fraction $f_{\mathrm{PEO}} \sim 0.15$ and a narrow molecular weight distribution (polydispersity $<1.1$ ) and exhibit bec morphology in the melt consisting of PEO spheres in a PI matrix (characterization data not shown). The synthesis of the nanostructured hybrids was performed by a PI- $b$-PEO directed sol-gel synthesis following a procedure described earlier. ${ }^{8,9}$ In this process, the hydrophilic PEO block is preferentially swollen by sol nanoparticles from a prehydrolyzed mixture of an organically modified ceramic precursor, (3-glycidyloxypropyl)trimethoxysilane (GLYMO), and aluminum sec-butoxide (the latter used as a catalyst and network hardener). The PEO domains act as nanoreactors for the further sol-gel synthesis of silica-type network structures. Different amounts of prehydrolyzed inorganic precursors (sol) were added to the block copolymer solution to obtain different morphologies. . $^{6,23}$

Alignment Experiments. After mixing the polymer with sol nanoparticles from the prehydrolyzed precursors in organic solvents (tetrahydrofuran/chloroform in a ratio of $1: 1$ by

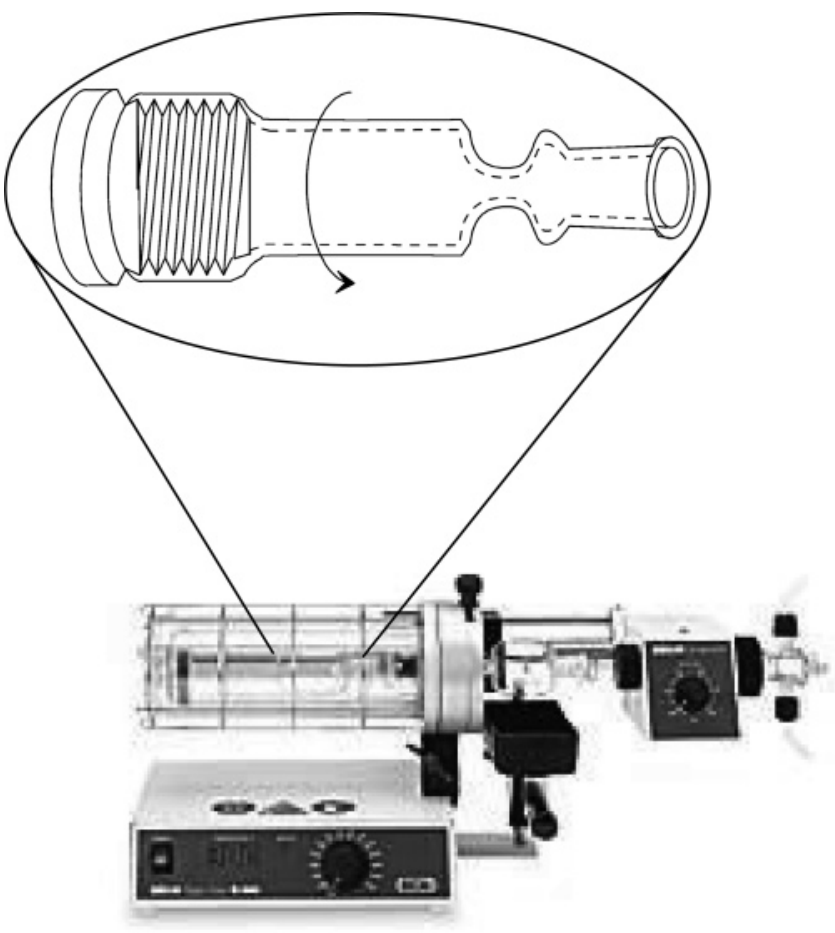

Figure 1. Schematic of the custom-built flow device with Teflon screw cap operated inside the Büchi evaporator. The arrow indicates the direction of flow.

weight), the solution was stirred in a vial for $1 \mathrm{~h}$ before drying/ casting. Static samples used as controls were prepared by transferring the solution from the vial in a Petri dish and heated at $50{ }^{\circ} \mathrm{C}$ for $1-2 \mathrm{~h}$ until all the solvents evaporated. Oriented films were formed by transferring the solution to a custom-built flow device (Figure 1) placed horizontally in a Büchi evaporator at $50{ }^{\circ} \mathrm{C}$ under dynamic vacuum, and the solution was rotated for 1-2 $\mathrm{h}$ until all the solvents evaporated off. The rotation speed of the cylinder is an important parameter in the setup for effective alignment of the nanostructure. It has to be chosen such that a stable flow profile is ensured during the course of film formation. A large value of rotation speed is likely to induce a larger orientation effect but at the same time leads to instabilities in the flow. A tradeoff is therefore needed to maximize the orientation. Based on these considerations, for an inner cylinder diameter of 25 $\mathrm{mm}$ of the flow device, a rotation speed of $\sim 15 \mathrm{rpm}$ was used which resulted in optimum orientation effects while ensuring a uniform film thickness. Further cross-linking of the gel and removal of the condensation products from both the control as well as the flow-aligned samples was achieved by additional heating at $130{ }^{\circ} \mathrm{C}$ in a vacuum oven, resulting in $0.5-1 \mathrm{~mm}$ thick nanostructured films.

Characterization. Small-angle X-ray scattering (SAXS) experiments were performed for characterization of the morphology and quantitative determination of the degree of order in the hybrid films. The data were collected on a Rigaku RUH3R copper rotating-anode $(\lambda=1.54 \AA)$ operated at $40 \mathrm{kV}$ and $50 \mathrm{~mA}$. X-rays were monochromatized with a nickel filter and focused using a set of orthogonal Franks mirrors. A home-built $1 \mathrm{~K} \mathrm{X} 1 \mathrm{~K}$ pixel 2-D CCD detector ${ }^{24}$ was used to record the scattering images. To determine the relative degree of ordering, scattering was performed in two mutually perpendicular directions for the static samples and three directions (flow, gradient, and neutral) for the oriented samples. Raw data were corrected for background and nonlinearitites in the detector. Beam divergence was not accounted for in the analysis. The order evaluation, therefore, provides an upper bound for full width at half-maximum (fwhm) and a lower bound for the calculated order parameters. 


\section{Results and Discussion}

Qualitative Observations. Three different hybrids were prepared under quiescent conditions by casting in a Petri dish for comparison with the flow aligned samples. Assuming negligible phase mixing between the PI (density $0.91 \mathrm{~g} / \mathrm{cm}^{3}$ ) and the inorganic/PEO microphases (density $1.4 \mathrm{~g} / \mathrm{cm}^{3}$ ), the volume fraction, $f$, of the inorganic/PEO domains in the three hybrids was estimated to be $0.36,0.49$, and $0.7 .{ }^{23}$ Azimuthally integrated scattering profiles of X-ray scattering intensity, $I$, vs the magnitude of the scattering vector, $q=$ $(4 \pi / \lambda) \sin \theta$, where $\lambda$ is the X-ray wavelength and $2 \theta$ is the scattering angle, for the three samples are shown in Figure $2 \mathrm{a}-\mathrm{c}$. The vertical dotted lines in Figure 2 indicate the best fitting lattices; i.e., in (a) and (c), they correspond to the expected peak positions for a hexagonal symmetry, while in (b), they correspond to a lamellar lattice. These morphologies are consistent with earlier results reported for this class of organic-inorganic hybrds. ${ }^{6,8,23}$ Figure $2 \mathrm{~d}$ shows the 2-D SAXS pattern of the hexagonal sample collected with the X-ray beam along the film normal and is representative for what is typically observed from hybrids synthesized under quiescent conditions. The pattern consists of a series of Debye-Scherrer rings, and the scattering intensity is nearly independent of the azimuthal scattering angle as expected from multigranular isotropic samples.

Similar results are observed in the 2-D SAXS patterns in Figure 3a for the inverse hexagonal sample prepared by solvent-casting in a Petri dish. Solvent-casting in a Petri dish is accompanied by a uniaxial compression perpendicular to the plane of the film as described and quantitatively analyzed previously. ${ }^{25}$ This explains the eccentricity $(\sim 22 \%)$ in the rings in the transverse SAXS pattern (Figure 3a) of the control sample. Besides the anisotropic shape, the patterns consist of rings with uniform intensity in both the normal and transverse directions, indicating the presence of randomly oriented grains. In contrast, 2-D SAXS patterns of the flow aligned inverse hexagonal sample in Figure $3 \mathrm{~b}$ show considerable sharpening of the spots in both the normal and neutral directions (see schematic in Figure $3 \mathrm{~b}$ for the definition of different directions). The distribution of scattering intensity now clearly depends on the azimuthal scattering angle indicative of a pronounced orientation effect resulting from casting of hybrids under flow. The simple flow geometry described above is thus capable of inducing a considerable orientation effect in bulk block copolymer-based organic-inorganic hybrids.

Quantitative Order Evaluation. As discussed above, the scattering intensity in the SAXS patterns shows a strong dependence on azimuthal scattering angle in the aligned samples. This distribution provides a basis for quantifying the degree of orientation. The orientation can mathematically be described with a 3 -dimensional probability distribution function $\rho(\phi)$ of the unit normal of the domains, $\mathbf{n}$, where $\phi$ is the solid angle of $\mathbf{n}$. The scattering pattern measured in a particular direction contains information about the slice of $\rho(\phi)$ in the plane perpendicular to the X-ray beam. This slice can be defined as a 2-dimensional probability distribution function $\omega(\theta)$, where $\theta$ is the azimuthal angle on the detector and can be estimated from the scattering
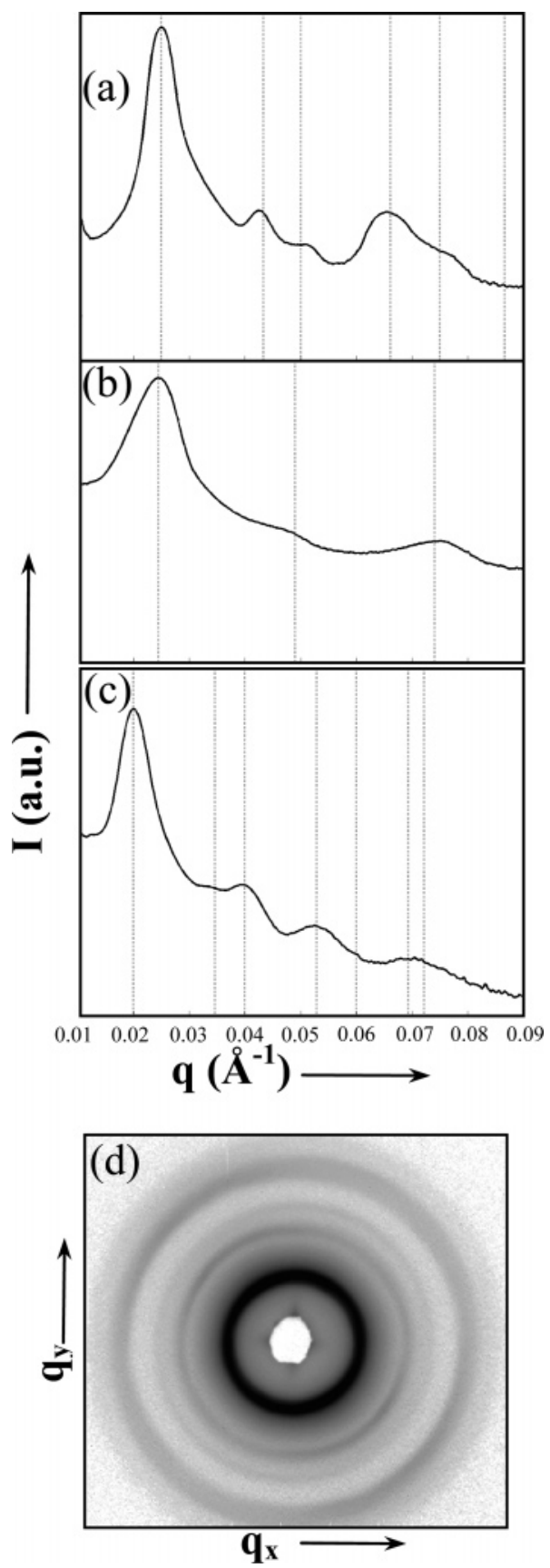

Figure 2. Azimuthally integrated scattering profile (X-ray intensity, $I$, vs scattering wave vector, $q$ ) measured for the hexagonal (a), lamellar (b), and inverse hexagonal (c) hybrids made by solvent-casting under quiescent conditions in a Petri dish. The vertical dotted lines in each plot correspond to the expected peaks from the respective symmetry group. (d) 2-D SAXS pattern collected with the X-ray beam incident along the plane normal of the film for a hexagonal hybrid.

intensity distribution $I(q, \theta)$ using the following equation: ${ }^{26,27}$

$$
\omega(\theta) \sin (\theta) \mathrm{d} \theta=\frac{\left[\int_{q_{2}}^{q_{1}} I(q, \theta) q^{2} \mathrm{~d} \theta\right] \sin (\theta) \mathrm{d} \theta}{\int_{0}^{\pi}\left[\int_{q_{2}}^{q_{1}} I(q, \theta) q^{2} \mathrm{~d} \theta\right] \sin (\theta) \mathrm{d} \theta}
$$

The complete distribution function is well sampled, within the most important planes, by the combination of the three 2-dimensional probability distribution functions measured in three orthogonal directions. The 2-dimensional distribution functions can be expanded 
(a)
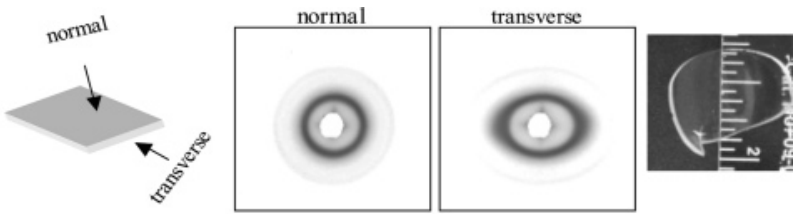

(b)
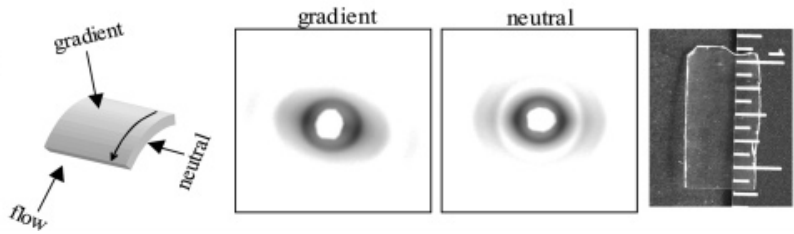

Figure 3. 2-D SAXS patterns along two orthogonal directions obtained from inverse hexagonal samples cast in quiescent conditions in a Petri dish (a) and in the flow device (b). The schematics on the left depict the definitions of different directions for these two processing conditions. The pictures on the right show pieces of the respective samples demonstrating that both films are clear and crack-free.

as a series of Legendre polynomials in which only the amplitudes of even coefficients are nonzero because of the symmetry of the structure factor: ${ }^{26}$

$$
\left\langle P_{2 n} \cos (\theta)\right\rangle=\int_{0}^{\pi} P_{2 n} \cos (\theta) \omega(\theta) \sin (\theta) \mathrm{d} \theta
$$

For situations of moderate order encountered in soft matter, $\left\langle P_{2} \cos (\theta)\right\rangle$ is often sufficient to describe the extent of orientation. $P_{2}$ has limits of $-1 / 2$ and 1 , corresponding to orientations of domains normal or parallel to the chosen director axis, respectively, and its value is 0 for randomly oriented domains (or singlecrystal orientation along the magic angle). For comparison of the orientation extent between samples measured with the X-ray beam along different directions, the director axis was chosen so that $\left\langle P_{2}\right\rangle$ has a value between 0 and 1 . This is realized by replacing $\theta$ with $\beta$ $=\theta-\theta_{0}$, where $\theta$ represents the azimuthal angle in the laboratory frame and $\theta_{0}$ is the angle between the order axis in the laboratory frame and the preferential orientation direction of the unit normals, $\mathbf{n}$, of the aligned domains in the sample. The final expression for $\left\langle P_{2}\right\rangle$ then reads as

$$
\left\langle P_{2} \cos (\beta)\right\rangle=\int_{0}^{\pi} w(\beta)\left[\frac{1}{2}\left(3 \cos ^{2}(\beta)-1\right)\right] \sin (\beta) \mathrm{d} \beta
$$

Figure 4 shows the detailed analysis for a flow-cast hexagonal sample consisting of hexagonally packed inorganic cylinders in an organic matrix. The raw data obtained along three orthogonal directions (flow, f; gradient, g; neutral, n) are shown in Figure 4a. The sharp six-spot pattern in the $q_{\mathrm{n}}-q_{\mathrm{g}}$ plane is quite remarkable and demonstrates the ability of this simple flow-cast technique to form quasi-single-crystalline domains in the direction of flow. The two-spot patterns in the $q_{\mathrm{f}}-q_{\mathrm{n}}$ and $q_{\mathrm{f}}-q_{\mathrm{g}}$ planes with reflections along $q_{\mathrm{n}}$ and $q_{\mathrm{g}}$, respectively, corroborate a uniaxial orientation of cylinders along the flow direction. As mentioned before, casting from solvents results in uniaxial compression in these hybrids. This compression is reduced but not completely eliminated in the flow-cast samples. For analysis of order in different directions, the raw data in Cartesian coordinates were first unfolded around the origin (primary beam) and converted to polar coordinates. Figure $4 \mathrm{~b}$ shows the unfolded data after correction for eccentricity in different directions. For order (a)

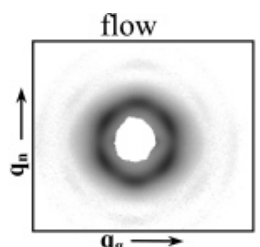

(b)
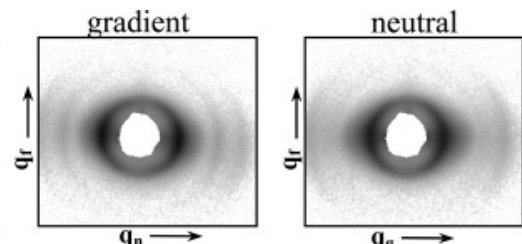

(c)
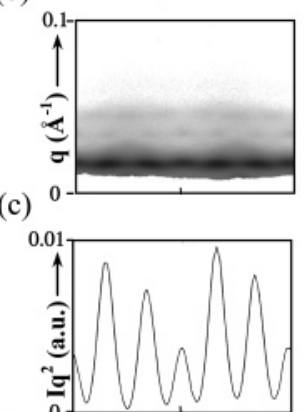

(d)
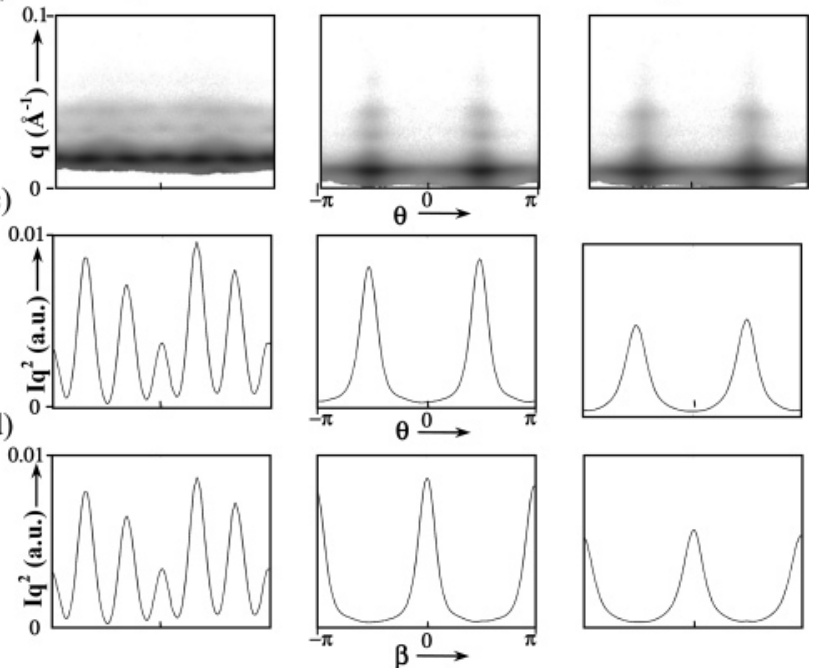

Figure 4. 2D SAXS data for three orthogonal incident X-ray beam directions and order evaluation process for a flow-cast hexagonal hybrid. Raw 2-D SAXS patterns for the three orthogonal directions - flow, gradient, and neutral-are shown in (a). The definition of the different directions is the same as in Figure 3b. The unfolded and eccentricity corrected patterns in polar coordinates are shown in (b). Integrations of the firstorder peak as a function of the azimuthal angle $\theta$ and adjusted azimuthal angle $\beta$ for the three directions are shown in (c) and (d), respectively.

anaylsis, the equations derived above are applied to the first-order peak in the scattering patterns. Figure $4 \mathrm{c}$ shows the X-ray intensity distribution as a function of azimuthal angle for the flow, gradient, and neutral directions. Finally, the azimuthal angle is adjusted so it aligns with the preferred director axis of the domains in the sample (Figure $4 \mathrm{~d}$ ), which restricts the computed order parameter to positive values as discussed above. Quantitative order analysis was performed for the patterns in the gradient and neutral directions, and the order parameters, $\left\langle P_{2}\right\rangle$, were determined to be 0.4 and 0.44 , respectively. The six-spot pattern in the flow direction excludes a simple determination of an order parameter. Instead, the average fwhm of individual peaks for scattering corresponding to the $q_{\mathrm{n}}-q_{\mathrm{g}}$ plane (flow direction) was determined to be $\sim 15^{\circ}$.

Similar analysis was performed for the lamellar as well as the inverse hexagonal samples prepared by flowcasting. Figure 5 shows scattering data and data processing for the order evaluation in the lamellar sample. The near-isotropic pattern in the $q_{\mathrm{f}}-q_{\mathrm{n}}$ plane in Figure $5 \mathrm{a}$ corresponding to the gradient direction results from the preferential orientation of lamellar domains parallel to the substrate. The sharp peaks along $q_{\mathrm{g}}$ in both $q_{\mathrm{n}}-q_{\mathrm{g}}$ and $q_{\mathrm{f}}-q_{\mathrm{g}}$ planes in Figure $5 \mathrm{a}$ corroborate this interpretation. Following the procedure described above for the hexagonal sample, the order parameters, $\left\langle P_{2}\right\rangle$, calculated from scattering data of the lamellar sample with the X-ray beam along the flow and neutral directions were determined to be 0.61 and 0.77 , respectively. Accordingly, the fwhm of individual peaks was determined to be as low as $8^{\circ}$ for scattering with 
(a)

(b)
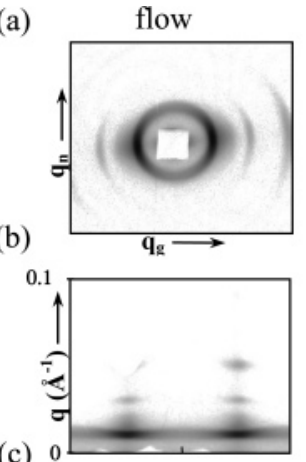

c)

(d)
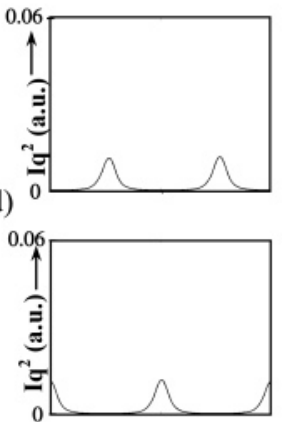

gradient

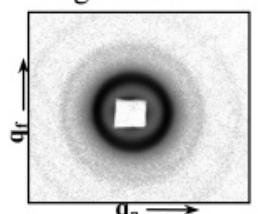

$\mathrm{q}_{\mathrm{n}}=$
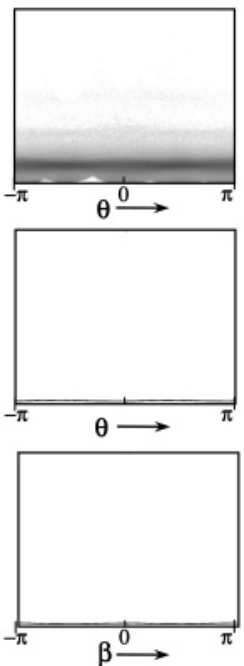

neutral

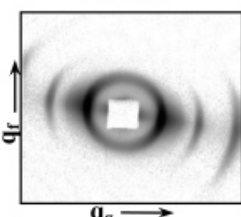

$\mathbf{q}_{\mathrm{g}}$
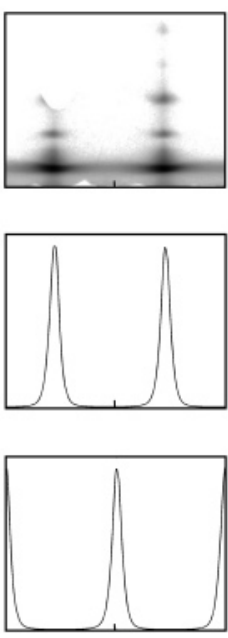

Figure 5. Order evaluation in flow-cast lamellar hybrids. Caption same as Figure 4. Please note the changed scale of the $y$-axes in (c) and (d) compared to Figures 4 and 6.

(a)

(b)
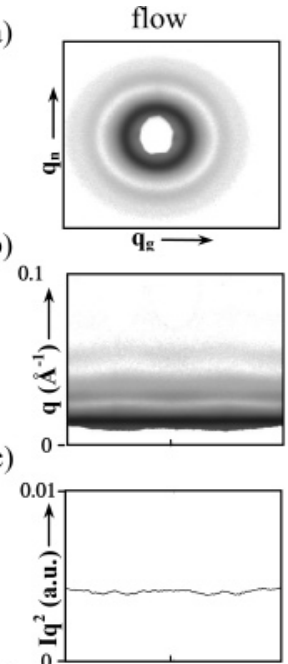

(d)
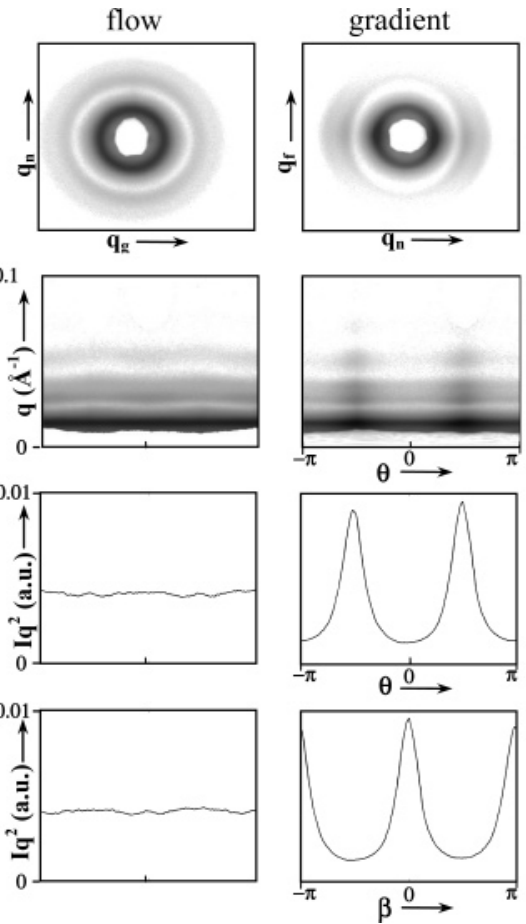

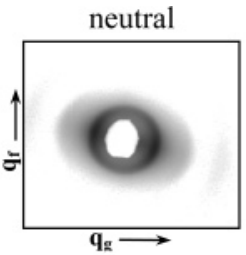

$\mathbf{q}_{\mathrm{g}}$
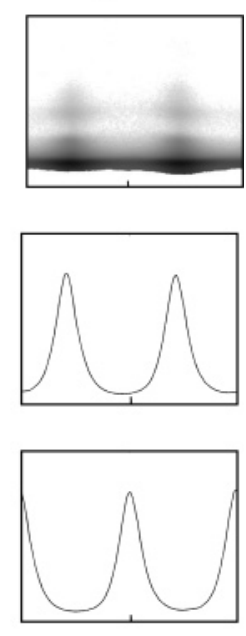

Figure 6. Order evaluation in flow-cast inverse hexagonal hybrids. Caption same as Figure 4.

X-ray beam along the neutral direction. This indicates a significant degree of orientation of lamellar planes parallel to the substrate that can be realized with this simple technique approaching values typically observed in smectic liquid crystal phases. ${ }^{28}$ For comparison, similar degrees of order have been reported for lamellar bulk block copolymer samples after $10 \mathrm{~h}$ of oscillatory shear. ${ }^{29}$

Finally, Figure 6 shows the scattering patterns for the flow-cast inverse hexagonal sample where the

inorganic forms the matrix for organic cylinders. As is evident from the near isotropic scattering pattern in the $q_{\mathrm{n}}-q_{\mathrm{g}}$ plane, in contrast to the case of the regular hexagonal sample, here flow is unable to achieve quasisingle-crystalline behavior. This can be attributed to the much higher inorganic fraction in the inverse phases, which is less compliant, making it more difficult to obtain alignment. However, even with such high inorganic loadings, flow-casting is able to provide a director axis for the domains indicated by the presence of sharp peaks along $q_{\mathrm{n}}$ in the $q_{\mathrm{f}}-q_{\mathrm{n}}$ plane and along $q_{\mathrm{g}}$ in the $q_{\mathrm{f}}-q_{\mathrm{g}}$ plane. The order parameters, $\left\langle P_{2}\right\rangle$, for these two directions were determined to be 0.37 and 0.4 , respectively, similar to the values obtained for the hexagonal sample. As expected, the fwhm of individual peaks in this case is also around $15^{\circ}$. Thus, for the inverse hexagonal sample, flow also provides a uniaxial director axis along the flow, but the overall morphology is multigranular when looked at along the flow direction.

\section{Conclusions}

A simple flow-casting technique for obtaining aligned block copolymer-based bulk polymer-inorganic hybrids has been demonstrated. Bulk hybrid nanostructured films with different morphologies were cast from organic solvents under flow and were found to be macroscopically aligned. Hexagonal samples with low inorganic content showed quasi-single-crystalline SAXS patterns when the X-ray beam was parallel to the flow direction. They exhibited considerable angular spread along the other two directions. Maximum orientation effects were found for the lamellar samples with order parameters for the lamellae parallel to the substrate as high as 0.77. After flow alignment, hexagonal hybrids with an inorganic matrix did not show quasi-single-crystalline patterns but exhibited similar order parameters around 0.4 for the orientation distribution of the cylinders along the flow direction as in the regular hexagonal samples. The present extension of the roll-casting technique ${ }^{21}$ thus holds promise for considerably improving the macroscopic order of sol-gel-derived bulk polymerinorganic hybrids. Alignment of these technologically relevant materials is vital for realizing their true potential, and development of simple techniques such as the one described in this work may hold the key for successful implementation of these functional materials in real applications. Furthermore, the orientation technique described herein is not limited to sol-gel-derived hybrids but rather should be applicable to any block copolymer-nanoparticle assemblies formed under evaporation of the solvent(s) which exclude conventional postsynthesis alignment methods.

Acknowledgment. The financial support of the National Science Foundation (Grant DMR- 0312913) is gratefully acknowledged. The work was further supported by the Cornell Center for Materials Research (CCMR), a Materials Research Science and Engineering Center of the National Science Foundation (DMR0079992), and DOE Grant DEFG02-97ER62443. We also thank Gil E. S. Toombes (Cornell University) for useful discussions and help with the analysis.

\section{References and Notes}

(1) Hamley, I. W. The Physics of Block Copolymers; Oxford University Press: London, 1998.

(2) Giannelis, E. P. Adv. Mater. 1996, 8, 29. 
(3) Kresge, C. T.; Leonowicz, M. E.; Roth, W. J.; Vartuli, J. C.; Beck, J. S. Nature (London) 1992, 359, 710.

(4) Beck, J. S.; Vartuli, J. C.; Roth, W. J.; Leonowicz, M. E.; Kresge, C. T.; Schmitt, K. D.; Chu, C. T.-W.; Olson, D. H.; Sheppard, E. W.; McCullen, S. B.; Higgins, J. B.; Schlenker, J. L. J. Am. Chem. Soc. 1992, 114, 10834.

(5) Antonietti, M.; Göltner, C. Angew. Chem. 1997, 109, 944.

(6) Templin, M.; Franck, A.; Du Chesne, A.; Leist, H.; Zhang, Y.; Ulrich, R.; Schadler, V.; Wiesner, U. Science 1997, 278, 1795.

(7) Zhao, D.; Feng, J.; Huo, Q.; Melosh, N.; Fredrickson, G. H.; Chmelka, B. F.; Stucky, G. D. Science 1998, 279, 548

(8) Simon, P. F. W.; Ulrich, R.; Spiess, H. W.; Wiesner, U. Chem. Mater. 2001, 13, 3464 .

(9) Jain, A.; Wiesner, U. Macromolecules 2004, 37, 5665.

(10) Thompson, R. B.; Ginzburg, V. V.; Matsen, M. W.; Anna, C. Balazs, A. C. Science 2001, 292, 2469.

(11) Finnefrock, A. C.; Ulrich, R.; Toombes, G. E. S.; Gruner, S. M.; Wiesner, U. J. Am. Chem. Soc. 2003, 125, 13084

(12) Jain, A.; Toombes, G. E. S.; Hall, L. M.; Mahajan, S.; Garcia, C. B. W.; Probst, W.; Gruner, S. M.; Wiesner, U. Angew. Chem., Int. Ed. 2005, 44, 1226.

(13) Koppi, K. A.; Tirrell, M.; Bates, F. S.; Almdal, K.; Colby, R. H. J. Phys. II 1992, 2, 1941.

(14) Leist, H.; Maring, D.; Thurn-Albrecht, T.; Wiesner, U. J. Chem. Phys. 1999, 110, 8225

(15) Chen, Z.-R.; Kornfield, J. A.; Smith, S. D.; Grothaus; T. J.; Satkowski, M. M. Science 1997, 277, 1248.

(16) Zryd, J. L.; Burghardt, W. R. Macromoloecules 1998, 31, 3656.
(17) Lu, Y.; Ganguli, R.; Drewien, C. A.; Anderson, M. T.; Brinker, C. J.; Gong, W.; Guo, Y.; Soyez, H.; Dunn, B.; Huang, M. H.; Zink, J. I. Nature (London) 1997, 389, 364.

(18) Melosh, N. A.; Davidson; Feng, P.; Pine, D. J.; Chmelka, B. F. J. Am. Chem. Soc. 2001, 123, 1240.

(19) Yang, P.; Zhao, D.; Chmelka, B. F.; Stucky, G. D. Chem Mater. 1998, 10, 2033.

(20) Brinker, C. J.; Lu, Y.; Sellinger, A.; Fan, H. Adv. Mater. 1999 , 11,579 .

(21) Albalak, R. J.; Thomas, E. L. J. Polym. Sci., Part B: Polym. Phys. 1993, 31, 37.

(22) Allgaier, J.; Poppe, A.; Willner, L.; Richter, D. Macromolecules 1997, 30, 1582

(23) Ulrich, R.; Du Chesne, A.; Templin, M.; Wiesner, U. Adv. Mater. 1999, 11, 141.

(24) Tate, M. W.; Gruner, S. M.; Eikenberry, E. F. Rev. Sci. Instrum. 1997, 68, 47.

(25) Finnefrock, A. C.; Ulrich, R.; Toombes, G. E. S.; Gruner, S. M.; Wiesner, U. J. Am. Chem. Soc. 2003, 125, 13084.

(26) Ward, I. M. Developments in Oriented Polymers-1; Elsevier Science: London, 1982.

(27) Ehlich, D.; Takenaka, M.; Hashimoto, T. Macromolecules 1993, 26,492 .

(28) De Gennes, P. G.; Prost, J. The Physics of Liquid Crystals, 2nd ed.; Oxford University Press: London, 1994.

(29) Zhang. Y.; Wiesner, U.; Yang, Y.; Pakula, T.; Spiess, H. W. Macromolecules 1996, 29, 5427.

MA0483930 